\title{
Involvement of endoplasmic reticulum stress-associated apoptosis in a heart failure model induced by chronic myocardial ischemia
}

\author{
WEI XIN ${ }^{1,2}$, XIAOYING LI ${ }^{1}$, XIAOCHUN LU ${ }^{1}, \mathrm{KUN} \mathrm{NIU}^{1}$ and JIMEI CAI ${ }^{1}$ \\ ${ }^{1}$ First Department of Geriatric Cardiology, Chinese PLA General Hospital, Beijing; \\ ${ }^{2}$ Medical College of Nankai University, Tianjin, P.R. China
}

Received October 1, 2010; Accepted November 25, 2010

DOI: $10.3892 / \mathrm{ijmm} .2011 .612$

\begin{abstract}
Apoptosis plays a critical role in the pathogenesis of chronic myocardial ischemia (CMI) and heart failure (HF). Endoplasmic reticulum stress (ERS) is one of the newly defined signaling pathways which initiate apoptosis. Previous studies have shown that ERS-associated apoptosis is involved in the pathogenesis of HF induced by pressure-overload and acute myocardial infarction. Also, in vitro experiments have proved that ischemia is a strong stimulus of ERS. This study aimed to demonstrate whether ERS-associated apoptosis is involved in the pathogenesis of CMI-induced HF. We established a HF model induced by CMI in mini pigs via placement of an ameroid constrictor around the proximal anterior descending branch of the left coronary artery (LAD). Furthermore, we used myocardial perfusion imaging, echocardiographic and hemodynamic measurements, hematoxylin-eosin staining, and terminal deoxynucleotidyl transferase-mediated DNA nick-end labeling staining to identify the existence of myocardial ischemia and cardiac dysfunction and of enhanced apoptosis in the ischemic heart. We performed immunohistochemistry, Western blot, and real-time PCR to analyze the hallmark of ERS glucose-regulated protein 78 (GRP78). The ERS-associated apoptotic pathways, CCAAT/enhancerbinding protein homologous protein (CHOP), caspase-12, and c-Jun $\mathrm{NH}_{2}$-terminal kinase 1 (JNK1) were also examined. We found that all three of these pathways were activated and that GRP78 protein and mRNA levels were significantly enhanced in the myocardium of HF mini pigs induced by CMI. These results suggest that ERS is present in the CMI-induced HF pig model, and that ERS-associated apoptosis is involved in the pathophysiology of HF induced by CMI.
\end{abstract}

Correspondence to: Professor Xiaoying Li, 28 Fuxing Road, First Department of Geriatric Cardiology, Chinese PLA General Hospital, Beijing 100853, P.R. China

E-mail: lixy@mx.cei.gov.cn.

Key words: chronic myocardial ischemia, heart failure, endoplasmic reticulum stress, apoptosis, glucose-regulated protein 78

\section{Introduction}

Heart failure (HF) remains a major cause of morbidity and mortality worldwide. In the population under the age of $65, \mathrm{HF}$ prevalence approaches $1 \%$ (1). Despite the fact that pharmacological and device-based treatments have improved survival, the 5-year mortality rate remains at an alarming 50\% in this population (2). Chronic myocardial ischemia (CMI) has become the most important cause of HF. In China, more than half of the patients with $\mathrm{HF}$ also have coronary heart disease (CHD) (3). These patients are suffering from CMI, leading to the further deterioration of cardiac function. The pathogenesis of CMI-induced HF is a chronic and complex process, which may involve abnormalities in energy metabolism, altered expression or function of contractile proteins, ventricular remodeling and defects in many organelles and cellular pathways (4).

Apoptosis, as a regulated, energy-dependent, programmed cell death mechanism has been reported to play a critical role in both CMI and HF $(5,6)$. The endoplasmic reticulum (ER) is recognized as an organelle that participates in the folding of secretory and membrane proteins $(7,8)$. Recent evidence suggests that another important function of ER is to regulate apoptosis $(9,10)$. Various stimuli, such as ER $\mathrm{Ca}^{2+}$ deprivation, ischemia, hypoxia, exposure to free radicals, oxidative stress, elevated protein synthesis and gene mutation, all of which can potentially cause ER dysfunction, are designated as ER stress (ERS) $(7,8,11)$. Several signaling pathways collectively called the unfolded protein response (UPR) are initiated to cope with ERS (12). One major pathway of the UPR is the upregulation of ER chaperones, such as the glucose-regulated protein $78 \mathrm{kDa}$ (GRP78), which can contribute to the repair of unfolded proteins (9). However, when ERS is excessive and prolonged, the initiation of apoptosis is promoted by transcriptional induction of the CCAAT/enhancer-binding protein (C/EBP) homologous protein (CHOP/GADD153) (13), the caspase-12 dependent pathway (14), and activation of the c-Jun $\mathrm{NH}_{2}$-terminal kinase 1 (JNK1)-dependent pathway (15).

It has been demonstrated that ERS-associated apoptosis is involved in the pathogenesis of HF induced by pressure-overload (16) and acute myocardial infarction (17). Also, in vitro experiment has proved that ischemia is a strong stimulus to ERS (18), but it is still not known whether ERS-associated apoptosis is involved in the pathogenesis of CMI-induced HF. In this study, we established HF models induced by CMI 
in mini pigs via placement of ameroid constrictors around the proximal anterior descending branch of the left coronary artery (LAD) to evaluate the role of ERS-associated apoptosis in this pathophysiological process.

\section{Materials and methods}

Mini pig models of chronic myocardial ischemia. Twelve male mini pigs (purchased from the animal experimental center of the Chinese PLA Hospital) weighing 24.5-27.5 kg were used in this study. The animals were randomized into 2 groups: 4 sham-operated animals (sham, $\mathrm{n}=4$ ) and 8 model animals (model, $\mathrm{n}=8$ ). Ameroid-induced progressive coronary occlusion was performed as described previously (19). Briefly, initial sedation was achieved with intramuscular injection of ketamine hydrochloride, diazepam and atropine. An ear vein was then cannulated for administration of an infusion of pentobarbital as needed to maintain anesthesia. The mini pig was intubated and ventilated. A left lateral thoracotomy was performed through the 4th intercostal space. After opening the pericardium, an ameroid constrictor $(2.5 \mathrm{~mm}$ of internal diameter, Research Instruments, USA) was implanted around the proximal LAD of the animals of the model group; this implantation was not performed for the animals of the sham group. Chronic myocardial ischemia was induced by progressive coronary artery stenosis with ameroid coronary constriction. The chest was then closed after a stable position of the constrictor was confirmed and antibiotics were given intramuscularly for the next three days. All protocols were approved by the Institutional Animal Care and Use Committee of the Chinese PLA General Hospital.

Myocardial perfusion imaging and single photon emission computed tomography (SPECT) scanning. Myocardial perfusion imaging was performed 4 weeks after ameroid placement using SPECT. One hour after intravenous injection of ${ }^{99 \mathrm{~m} T c}-\mathrm{MIBI}$, tomographic imaging was acquired using a rotating gamma camera equipped with a high-resolution collimator. Reconstructions were performed by an experienced expert in nuclear medicine who was blinded to the treatment group. The imaging data were processed and analyzed by automatic computerized planimetry. The size of the defect was calculated using a threshold of $50 \%$. The extent of the perfusion defect was quantified as a percentage of the entire left ventricular mass on a polar map.

Echocardiographic assessment. Transthoracic echocardiograms were performed before and 4 weeks after ameroid placement, using the Sequoia 2512 cardiovascular ultrasound system (Siemens, Germany) with a $3-\mathrm{MHz}$ transducer. Serial echocardiographic data were collected in a blinded fashion. The Teichholz method was employed to calculate left ventricular (LV) volumes and ejection fraction (LVEF). Parasternal long and short axes views were used to measure the following parameters: LV diameters at end-diastole (LVDd) and end-systole (LVSd), LVEF and the ratio between early and atrial peak filling velocities (Ev/Av). The average of at least 3 consecutive cardiac cycles was used for each measurement.

Hemodynamic measurements. Four weeks after ameroid placement, a 7F Swan-Ganz catheter (Baxter Healthcare Corp.,
Irvine, USA) was placed to the pulmonary artery via the right external jugular vein to measure the mean pulmonary arterial pressure (mPAP) and the pulmonary capillary wedge pressure (PCWP). A common carotid artery catheter (RM6240, Instruments Inc., Chengdu, China) was positioned in the LV cavity to collect the following parameters: LV systolic (LVSP) and end diastolic pressure (LVEDP), maximal rate of rise (+dp/ $\left.\mathrm{dt}_{\max }\right)$ and decline $\left(-\mathrm{dp} / \mathrm{dt}_{\max }\right)$ of LV pressure and the heart rate (HR).

Tissue collection and preparation. Animals were sacrificed by intravenous injection of potassium chloride after hemodynamic measurements. The heart was harvested and the left ventricle was cut into transverse sections of $\sim 1 \mathrm{~cm}$ thickness from apex to base. The segments of the anterior wall of the LV, an ischemic area supplied by the LAD were fixed overnight in $10 \%$ formalin and embedded in paraffin for histological analysis. In addition, parts of the segments were snap-frozen in liquid nitrogen and stored at $-80^{\circ} \mathrm{C}$ for Western blot and real-time PCR analysis.

Histopathology, TUNEL, and immunohistochemistry (IHC) staining. Myocardium segments embedded in paraffin were sectioned at $6 \mu \mathrm{m}$ and studied with hematoxylin-eosin stain, a terminal deoxynucleotidyl transferase (TDT) -mediated DNA nick-end labeling (TUNEL) assay kit, and IHC. The sections were examined using light microscopy and analyzed with Leica QWin software.

Apoptosis assessment was conducted using a commercially available TUNEL assay kit (Promega, USA). Briefly, sections were deparaffinized, digested with $20 \mathrm{mg} / \mathrm{ml}$ proteinase $\mathrm{K}$, at room temperature for $15 \mathrm{~min}$, and soaked in PBS for 5 min. Each section was covered with TDT enzyme solution and incubated for $1 \mathrm{~h}$ at $37^{\circ} \mathrm{C}$ in a humidified chamber. The sections were immersed in stop buffer to terminate the enzymatic reaction, and then gently rinsed with PBS. Streptavidin-horseradish peroxidase (HRP) solution was then applied to each section and incubated at room temperature for $30 \mathrm{~min}$ in the darkness. The slides were washed in PBS, exposed to 3,3'-diaminobenzidine (DAB) (Golden Bridge Biotechnology, Beijing, China) for 5-7 min, rinsed in water and counterstained with hematoxylin. Finally, the sections were examined using light microscopy.

The tissue expression of GRP78 was assessed immunohistochemically using an anti-GRP78 antibody (Santa Cruz, USA). After deparaffinization, endogenous peroxidase activity was quenched with $30 \%$ methanol and $0.3 \%$ hydrogen peroxide in PBS. The slides were then boiled in citrate buffer in a microwave. After blocking non-specific binding with 5\% BSA, the slides were incubated with primary antibodies overnight at $4^{\circ} \mathrm{C}$ (1:400 dilution of anti-GRP78). The following day, the sections were thoroughly washed in PBS and incubated with a peroxidase-conjugated polymer that carries antibodies to goat (1:200) immunoglobulin (Golden Bridge Biotechnology) for $30 \mathrm{~min}$. After rinsing with PBS, the sections were exposed to $\mathrm{DAB}$ for $7 \mathrm{~min}$. The slides were rinsed in water and counterstained with hematoxylin.

Western blot analysis. Protein from the myocardium of sham and model mini pigs was extracted for Western blot analysis. 
Table I. The sequence for each primer and product length.

\begin{tabular}{llr}
\hline & \multicolumn{1}{c}{ Sequence } & Length \\
\hline GRP78 & F: 5'-CGAGGAGGAGGACAAGAAGG-3' & $302 \mathrm{bp}$ \\
& R: 5'-CACCTTGAACGGCAAGAACT-3' & $195 \mathrm{bp}$ \\
JNK1 & F: 5'-CAAGCACCTTCACTCTGC-3' & $372 \mathrm{bp}$ \\
& R: 5'-TTCTTTGTAGCCCATCCC-3' & \\
\hline
\end{tabular}

The tissue samples were homogenized and centrifuged at $7000 \mathrm{x} \mathrm{g}$ for $15 \mathrm{~min}$ at $4^{\circ} \mathrm{C}$ The supernatants were used as protein samples. The Bradford assay was used to determine each sample's protein concentration and Western blot analysis was performed with a $5 \%$ acrylamide stacking gel and a $14 \%$ acrylamide resolving gel. Transfer of proteins from the gel to nitrocellulose membranes was performed for $50 \mathrm{~min}$ at $120 \mathrm{~V}$. Non-specific protein binding to the nitrocellulose membrane was reduced by pre-incubating the membrane with blocking buffer for $2 \mathrm{~h}$ at room temperature. This step was followed by incubation with the monoclonal antibodies, anti- $\beta$-actin (1:1000 diluted in blocking buffer, Golden Bridge Biotechnology), anti-GRP78 (1:500), anti-CHOP (1:500, Santa Cruz Biotechnology), anti-caspase-12, (1:1000, Santa Cruz Biotechnology), anti-JNK1 and anti-phosphorylated-JNK1 (pJNK1, 1:2000, Santa Cruz Biotechnology) overnight at $4{ }^{\circ} \mathrm{C}$. After incubation with a secondary antibody for $1 \mathrm{~h}$ at room temperature, the signal was detected by enzyme linked chemiluminescence (ECL kit, BioSciences Inc., NY, USA).

Real-time PCR analysis. Total RNA was extracted from the myocardium of sham and model animals using TRIzol reagent (Invitrogen, USA) according to the manufacturer's instructions. The RNA was quantified by UV spectrophotometry at $260 / 280 \mathrm{~nm}$ and diluted to $1 \mathrm{mg} / \mathrm{ml}$ in diethyl pyrocarbonate (DEPC)-treated water.

First-strand cDNA was synthesized in a $40 \mu \mathrm{l}$ total volume by using oligo(dT) 12-18 primer and the Moloney murine leukemia virus (MMLV) reverse transcriptase (Promega). Briefly, reverse transcription was performed in a $40 \mu \mathrm{l}$ volume mixture made up of $6 \mu 1$ total RNA, $2 \mu 1$ oligo(dT), $8 \mu 1$ reverse-transcription buffer, $2 \mu 12.5 \mathrm{mM}$ dNTP mixture, $2 \mu 1$ MMLV reverse transcriptase, and $20 \mu 1$ DEPC-treated water. The mixture was incubated at $37^{\circ} \mathrm{C}$ for $1 \mathrm{~h}$ and then heated at $95^{\circ} \mathrm{C}$ for $5 \mathrm{~min}$. The cDNA was stored at $-20^{\circ} \mathrm{C}$ for use.

Primer sequences and product length of each gene are presented in Table I. Real-time PCR was carried out using the real-time detection system (ABI Prism 7500, USA) with a real-time PCR master mix (Toyobo, Japan) that contains SYBR-Green I as the fluorescent dye. The protocol was followed according to the manufacturer's instructions. A $50 \mu \mathrm{l}$ volume mixture was made up from $1 \mu \mathrm{l}$ sense primer $(10 \mu \mathrm{M})$, $1 \mu \mathrm{l}$ antisense primer $(10 \mu \mathrm{M}), 25 \mu \mathrm{l}$ real-time PCR master mix, $18 \mu \mathrm{l}$ DEPC-treated water, and $5 \mu \mathrm{l}$ cDNA. The cycling conditions were $95^{\circ} \mathrm{C}$ for $3 \mathrm{~min}$, followed by 40 cycles at $94^{\circ} \mathrm{C}$ for $30 \mathrm{sec}, 60^{\circ} \mathrm{C}$ for $30 \mathrm{sec}$, and $72^{\circ} \mathrm{C}$ for $60 \mathrm{sec}$. For quantifi- cation, the GRP78 and JNK1 gene mRNAs were normalized to the mRNA of the internal standard gene glyceraldehyde-3phosphate dehydrogenase (GAPDH).

Statistics. Results are expressed as the mean value \pm standard deviation (SD). The individual groups were tested for differences by using the independent samples t-test. A probability value $(\mathrm{P})$ of $\leq 0.05$ was considered to be statistically significant. Statistical analysis was performed using SPSS 13.0 software (SPSS Inc., Chicago, USA).

\section{Results}

Establishement and evaluation of the HF model induced by CMI. Six animals in the model group survived while the other 2 died during the procedure of ameroid implantation (1 died of ventricular fibrillation, the other died of major hemorrhage caused by vessel injury). All animals of the sham group survived the surgery. Three weeks after ameroid placement, some animals in model group showed symptoms of tachypnea, hypokinesia and poor ingestion, indicating the appearance of $\mathrm{HF}$.

Perfusion defect. Four weeks after ameroid placement, SPECT demonstrated a significant myocardial perfusion defect in the LAD supplied territories (ventricular septum, apex and frontal wall of LV) in the model group (Fig. 1B and D). Presented as a percentage of the entire left ventricular mass on a polar map, the perfusion defect of the model group was $41.4 \pm 15.2 \%$, while no significant myocardial perfusion defect was observed in animals of the sham group (Fig. 1A, C and E).

Cardiac dysfunction. Four weeks after ameroid constrictor placement, the cardiac functions of animals in each group were evaluated by echocardiography and hemodynamic measurements. Compared to the echocardiographic data before ameroid placement, the LVEF and Ev/Av were significantly reduced $(\mathrm{P}<0.05)$, indicating severe systolic and diastolic dysfunction in the model group. On the other hand, LVDd and LVSd were significantly increased, suggesting an enlargement of the LV in the model group $(\mathrm{P}<0.05$, Table II). Hemodynamic data of animals 4 weeks after ameroid constrictor placement are shown in Table III. Compared with the sham group, LVSP, $+\mathrm{dp} / \mathrm{dt}_{\text {max }}$ and $-\mathrm{dp}_{\mathrm{dt}} \mathrm{dt}_{\max }$ were significantly reduced $(\mathrm{P}<0.05)$; while LVEDP, mPAP and PCWP $(\mathrm{P}<0.05)$ were siginificantly increased, also suggesting a severe cardiac dysfunction in the model group. 
A

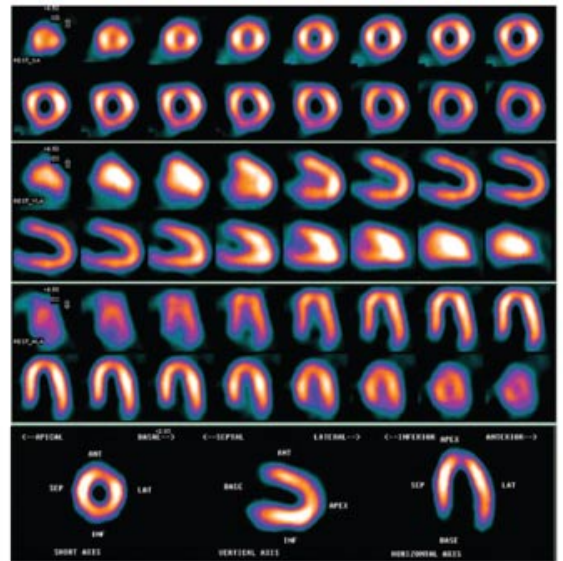

C

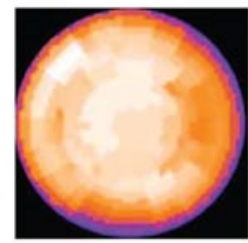

D

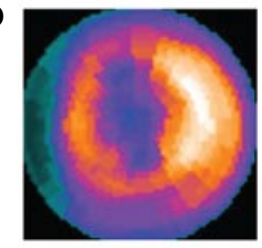

B

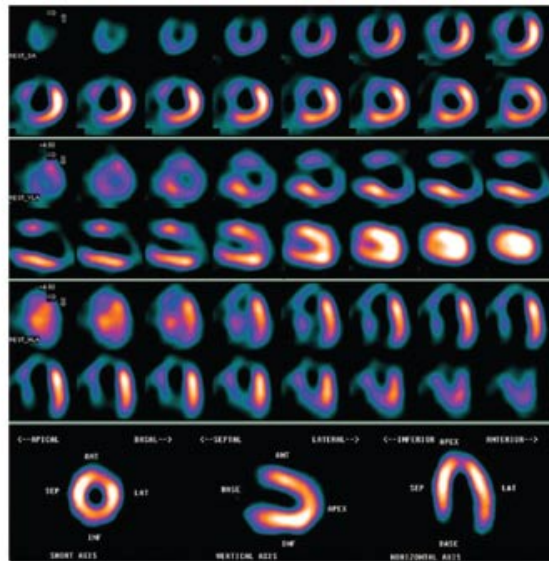

$\mathbf{E}$

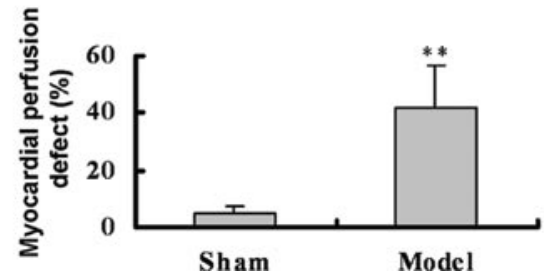

Figure 1. Placement of an ameroid constrictor induced a myocardial perfusion defect in LAD-supplied territories of the model group. (A) and (B) Representative tomographic images of myocardial perfusion in the sham and model groups by SPECT scanning. (C) and (D) Representative polar maps of myocardial perfusion in the sham and model groups. (E) Semi-quantitative analysis of the myocardial perfusion defect in both groups. The extent of the perfusion defect was quantified as a percentage of the entire left ventricular mass on a polar map; $n=4$ in the sham group, $n=6$ in the model group. Compared to sham group, ${ }^{* *} \mathrm{P}<0.01$.
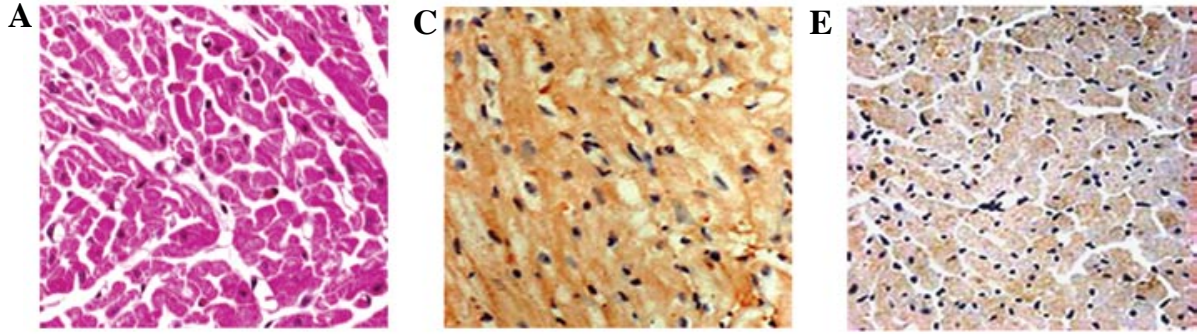

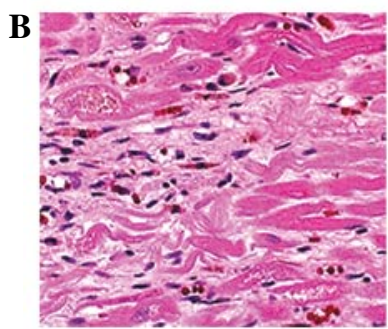

G

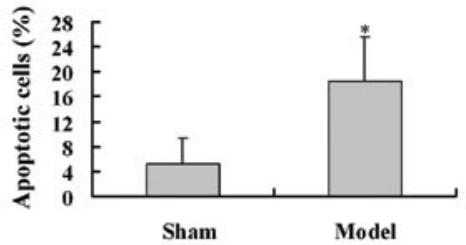

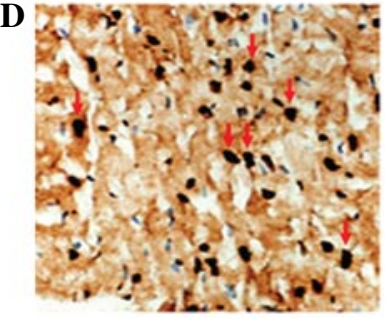

$\mathbf{H}$

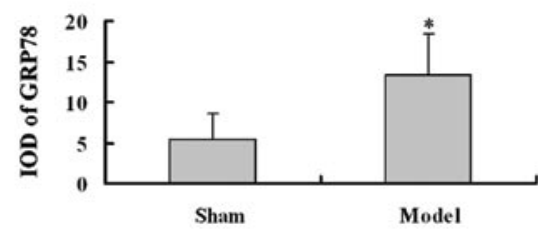

Figure 2. (A) and (B) Hematoxylin-eosin staining of the myocardium of the sham and model groups. (C) and (D) TUNEL staining of apoptotic bodies (pointed by arrows) of the myocardium in the sham and model groups. (E) and (F), IHC staining for GRP78 in the myocardium of the sham and model groups. (G) and (H) Semi-quantitative analysis of the percentages of the apoptotic cells and GRP78 protein levels in the myocardium of both groups; $n=4$ in the sham group, $\mathrm{n}=6$ in the model group. Compared to the sham group, ${ }^{*} \mathrm{P}<0.05$. Bar, $50 \mu \mathrm{m}$.

Histopathology. Hematoxylin-eosin staining (Fig. 2A and B), demonstrated that ischemic cardiac muscle fibers were disordered, and many of them were collapsed. The ischemic myocardium showed fibrosis and extensive focal coalescent 
Table II. Echocardiographic data before and 4 weeks after ameroid placement in model group.

Before ameroid placement $(n=6)$

After ameroid placement $(\mathrm{n}=6)$

\begin{tabular}{lcr}
\hline LVDd $(\mathrm{mm})$ & $31.3 \pm 4.9$ & $37.2 \pm 5.2^{\mathrm{a}}$ \\
LVSd $(\mathrm{mm})$ & $19.7 \pm 4.6$ & $29.5 \pm 6.7^{\mathrm{a}}$ \\
LVEF $(\%)$ & $65.3 \pm 8.3$ & $42.4 \pm 10.1^{\mathrm{a}}$ \\
EV/Av & $1.21 \pm 0.19$ & $0.79 \pm 0.18^{\mathrm{a}}$
\end{tabular}

LVDd, LV diameters at end-diastole; LVSd, LV diameters at end-systole; LVEF, LV ejection fraction; Ev/Av, the ratio between early and atrial peak filling velocities. Compared to model group before ameroid placement, ${ }^{\mathrm{a}} \mathrm{P}<0.05$.

Table III. Hemodynamic data 4 weeks after ameroid placement in each group.

\begin{tabular}{|c|c|c|c|c|c|c|c|}
\hline & $\begin{array}{c}\text { mPAP } \\
(\mathrm{mmHg})\end{array}$ & $\begin{array}{l}\text { PCWP } \\
(\mathrm{mmHg})\end{array}$ & $\begin{array}{c}\text { LVSP } \\
(\mathrm{mmHg})\end{array}$ & $\begin{array}{l}\text { LVEDP } \\
(\mathrm{mmHg})\end{array}$ & $\begin{array}{c}+\mathrm{dp} / \mathrm{dt}_{\max } \\
(\mathrm{mmHg} / \mathrm{sec})\end{array}$ & $\begin{array}{c}-\mathrm{dp} / \mathrm{dt}_{\max } \\
(\mathrm{mmHg} / \mathrm{sec})\end{array}$ & $\begin{array}{c}\mathrm{HR} \\
(\mathrm{bpm})\end{array}$ \\
\hline Sham $(n=4)$ & $16.8 \pm 3.1$ & $7.1 \pm 2.2$ & $119.3 \pm 13.2$ & $7.6 \pm 1.8$ & $2799.1 \pm 388.4$ & $1424.3 \pm 274.9$ & $88.2 \pm 9.7$ \\
\hline Model $(n=6)$ & $25.6 \pm 7.3^{\mathrm{a}}$ & $14.2 \pm 5.1^{\mathrm{a}}$ & $90.8 \pm 13.1^{\mathrm{a}}$ & $22.8 \pm 6.0^{\mathrm{a}}$ & $1899.3 \pm 521.4^{\mathrm{a}}$ & $868.5 \pm 318.6^{\mathrm{a}}$ & $102.1 \pm 15.7$ \\
\hline
\end{tabular}

mPAP, mean pulmonary arterial pressure; PCWP, pulmonary capillary wedge pressure; LVSP, LV systolic pressure; LVEDP, LV end-diastolic pressure; $+\mathrm{dp} / \mathrm{dt}_{\max }$, maximal rate of rise of $\mathrm{LV}$ pressure; $-\mathrm{dp} / \mathrm{dt}_{\max }$, maximal rate of decline of $\mathrm{LV}$ pressure; $\mathrm{HR}$, heart rate. Compared to sham group, ${ }^{\mathrm{a}} \mathrm{P}<0.05$.

A
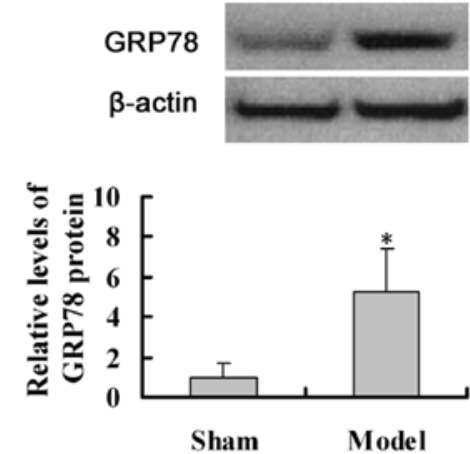

C Procaspase-12 Cleaved
caspase-12

$\beta$-actin

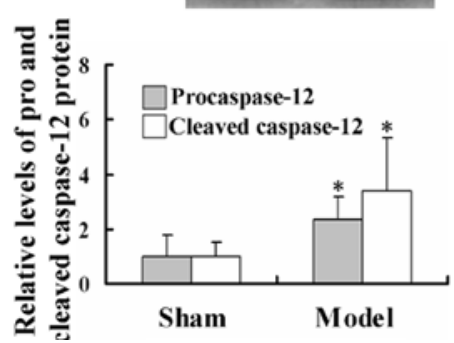

B

CHOP

$\beta$-actin
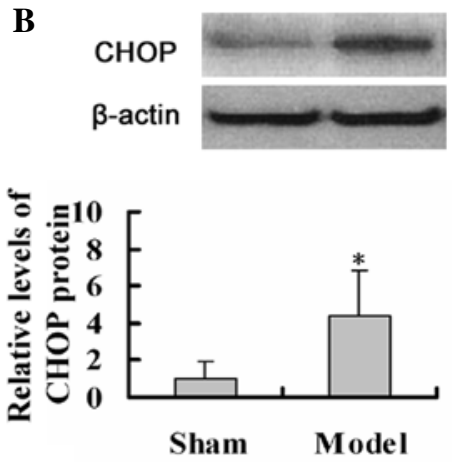

D P-JNK1

Total JNK1

$\beta$-actin

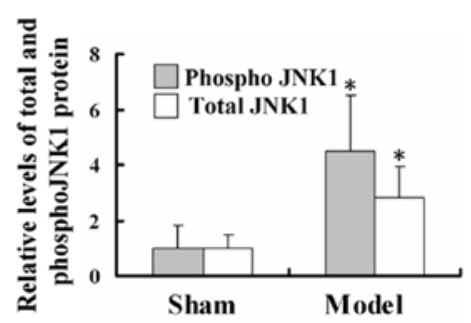

Figure 3. Immunoblot analyses for (A) GRP78, (B) CHOP, (C) caspase-12, and (D) JNK1 in the myocardium of sham and model groups. The upper trace of each panel shows representative blots of the respective protein in the myocardium of sham (left band) and model (right band) animals. The bar graphs summarize the immunoblot data. Densitometric results are expressed as the mean \pm SD of the fold induction of the band density obtained from the myocardium of model animals compared to that of sham animals; $n=4$ in the sham group, $n=6$ in the model group. Compared to the sham group, $P \leq 0.05$.

areas of ischemic cardiomyocyte degeneration. Micro myocardial infarction and necrosis could also be detected. The above results certified that ameroid constrictor placement was an effective way to induce HF by causing CMI.
Localization of apoptosis by the TUNEL assay and IHC analysis of GRP78 distribution. To assess whether CMI results in apoptotic cell death in the ischemic myocardium, the tissue sections were labeled with an in situ TUNEL assay. 


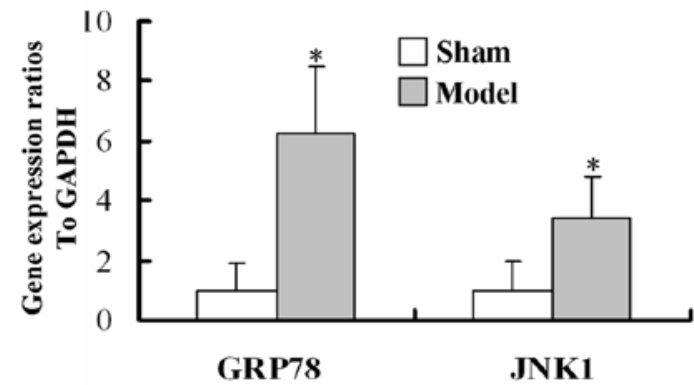

Figure 4. GRP78 and JNK1 mRNA expression analysis in myocardium of sham and model groups by real-time RT-PCR. Values represent the ratio to GAPDH mRNA that is arbitrarily defined as 1 . Data are shown as mean $\pm \mathrm{SD}$; $\mathrm{n}=4$ in sham group, $\mathrm{n}=6$ in model group. Compared with sham group, ${ }^{*} \mathrm{P}<0.05$.

Apoptosis was observed in both the cardiomyocytes and endothelium of the ischemic myocardium in the model group (Fig. 2D). Estimation of cardiac apoptosis revealed a nearly 4-fold increase in TUNEL-positive nuclei in the myocardium of the model group (Fig. 2G). As can be seen in Fig. 2F, ICH studies showed that GRP78 was abundantly expressed in the myocardium of the model group. In contrast, the myocardium of the sham group exhibited modest or weak immunoreactivity for this molecule (Fig. 2E and H). We also found that in the myocardium of the sham group, the increase in GRP78-positive cells paralleled the increase of apoptotic cells (Fig. 2F).

Western blot analysis of GRP78, CHOP, caspase-12 and $J N K 1$. Western blot analysis (Fig. 3) demonstrated significant increases of GRP78, CHOP, pro and cleaved caspase-12, total JNK1and pJNK1 levels in the myocardium of model group animals $(\mathrm{P}<0.05)$. The results indicate that $\mathrm{CHOP}$ was induced, and caspase-12 and JNK1 pathways were activated in the myocardium of model group animals.

Real-time RT-PCR analysis of GRP78 and JNK1 expression. Changes in GRP78 and JNK1 mRNA expressions were quantified by real-time RT-PCR of RNA from the myocardium of sham and model animals (Fig. 4). GRP78 and JNK1 mRNA expressions were significantly increased in the myocardium of model animals $(\mathrm{P}<0.05)$, paralleling their enhanced protein expression.

\section{Discussion}

It has been demonstrated that the ERS pathways are activated during the pathogenesis of pressure-overload or AMI-induced HF, although this conclusion has been based on observations in in vitro or in small animal in vivo models (20-23). In the current study, we established a large animal model of CMI-induced HF by ameroid constrictor placement in mini pig, and we performed echocardiographic and hemodynamic measurements to confirm the existence of cardiac dysfunction and myocardial hematoxylin-eosin staining to identify the histological changes of CMI and HF. The results of TUNEL staining indicated that more cardiomyocytes and endothelial cells were apoptotic in the myocardium of model animals than in that of sham animals. We demonstrated that GRP78 expression is enhanced which is in parallel to the increase in apoptotic cells. Protein and mRNA levels of three hallmarks and their activated forms of ERS-induced apoptosis: CHOP, pro and cleaved caspase-12, total JNK1 and pJNK1, were increased. These findings suggest that ERS was induced in our experimental model, and ERS-associated apoptosis pathways were activated. To our knowledge, this is the first study that implicates that ERS-associated apoptosis in the pathogenesis of CMI-induced HF in large animals.

Ameroid constrictor is a device containing an outer ring of stainless steel and an inner ring of casein, which swells as it absorbs tissue fluid, causing gradual occlusion of blood flow in the LAD. Besides, its mechanical pressure can lead to the injury of endothelial cells resulting in subsequent thrombosis that is also involved in the occlusion of the blood flow (24). After implantation with the proper size of ameroid constrictor, it usually takes 3 weeks to achieve vessel occlusion (24). Because, similarly to humans, the coronary arteries' ability to form collateralizations in the pig is limited, persistent ischemia can occur after ameroid implantation (19). Also, LADs are essential for the blood supply of the heart; therefore, placement of the ameroid around the proximal LAD can cause more severe myocardial ischemia than occlusion of other branches and is more likely to cause HF. Four weeks after ameroid placement, SPECT demonstrated an irreversible perfusion defect in the LAD-dependent myocardium of the model group. Echocardiographic and hemodynamic parameters showed a significant reduction in both systolic and diastolic cardiac function, together with the characteristic changes of the ischemic myocardial histopathology indicated by hematoxylin-eosin staining, suggesting that continuing myocardial ischemia caused HF in the model animals.

It has been suggested that apoptosis plays an important role in the pathogenesis of both CMI and HF $(5,6)$. Ischemia induces myocardial apoptosis, which results in loss of cardiomyocytes, leading to the impairment of cardiac systolic and diastolic functions. A few studies have also demonstrated that ischemia, along with the deprivation of oxygen, nutrition and energy supply, lead to the activation of the ERS pathways in cultured cardiomyocytes or hearts in vitro $(17,18,20-23)$. These results and the increasing incidence of CMI-related HF prompted us to examine whether ERS-specific apoptotic signaling is involved in the heart of CMI-induced HF.

GRP78, also referred to as immunoglobulin heavy chain binding protein (Bip), is a central regulator of ER function due to its roles in protein folding and assembly, targeting misfolded protein for degradation, $\mathrm{ER} \mathrm{Ca}^{2+}$ binding, and controlling the activation of transmembrane ERS sensors. Induction of GRP78 has been widely used as a marker for ERS and the onset of UPR (25). Furthermore, GRP78 serves as a master modulator for the UPR network by binding to ERS sensors such as, protein kinase R-like ER kinase (PERK), inositol requiring 1 (IRE1), and activating transcription factor 6 (ATF6) and by inhibiting their activation (25). Therefore, in this study, we used GRP78 as a marker of ERS and UPR pathways.

CHOP, caspase-12 and JNK1 are three well-defined apoptotic pathways related to ERS. CHOP belongs to the C/EBP family of transcription factors and is transcriptionally induced during the development of ERS by ATF6 and IRE1 signaling $(26,27)$. Overexpression of CHOP promotes apoptosis, and 
CHOP deficiency can protect cells from ERS-induced apoptosis, suggesting that $\mathrm{CHOP}$ is involved in the process of cell death caused by ERS, and that overexpression of CHOP sensitized the cells to ERS via down-regulation of Bcl-2 expression $(14,28,29)$. Caspase-12-mediated apoptosis is a specific apoptotic pathway of the ER, and apoptosis that has occurred as a result of membrane- or mitochondrial-targeted signals do not activate it (30). ERS leads to an increase in cytosolic calcium levels that induce the activation of m-calpain. Activated $\mathrm{m}$-calpain cleaves Bcl-xL and proteolytically activates caspase12. Cleaved caspase-12 reportedly activates caspase- 9 , followed by activation of caspase-3 (31). The caspase-12 knockout mice exhibit resistance to ERS, suggesting that caspase-12 also plays a role in the process of cell death caused by ERS. JNK1 was activated during ERS through phosphorylation mediated by the formation of the IRE1-tumor necrosis factor receptor associated factor 2-apoptosis signal-regulating kinase 1 complex (32). Activation of JNK1 is a common response to many forms of stress and is known to influence the cell-death machinery through the regulation of BCL2 family proteins (33). Phosphorylation of BCL2 by activated JNK1, which occurs primarily at the ER, suppresses the anti-apoptotic activity of $\mathrm{Bcl}-2$. In the present study, the increased induction of GRP78, CHOP, pro and cleaved caspase-12, total JNK1 and pJNK1 in myocardium of model animals were observed, paralleling that of the increase in apoptotic cells, indicating the activation of ERS and associated apoptotic pathways in the pathogenesis of CMI-induced HF.

In summary, this study provides the first evidence of the GRP78 increase and of the activation of the three pathways of ERS-induced apoptosis in the myocardium of large HF animals induced by CMI. Our findings suggest that ER-initiated apoptosis may contribute to ischemic cardiomyopathy and related $\mathrm{HF}$, and further investigation of the intracellular signaling and the weight of ERS-induced apoptosis in the failed heart induced by CMI will be required.

\section{Acknowledgements}

This study was supported by the National Basic Research Development Program of China, namely '973' Program (No. 2007CB512004), and the National Science Foundation of China (No. 30600236 and 30770900).

\section{References}

1. Lloyd-Jones D, Adams RJ, Brown TM, et al: Heart disease and stroke statistics - 2010 update: a report from the American Heart Association. Circulation 121: e46-e215, 2010.

2. Jessup M and Brozena S: Heart failure. N Engl J Med 348: 2007-2018, 2003.

3. Jiang $\mathrm{H}$ and $\mathrm{Ge} \mathrm{J}$ : Epidemiology and clinical management of cardiomyopathies and heart failure in China. Heart 95: 1727-1731, 2009.

4. Braunwald E and Bristow MR: Congestive heart failure: fifty years of progress. Circulation 102: IV14-IV23, 2000.

5. Dorn GW II: Apoptotic and non-apoptotic programmed cardiomyocyte death in ventricular remodelling. Cardiovasc Res 81: 465-473, 2009.

6. Lee Y and Gustafsson AB: Role of apoptosis in cardiovascular disease. Apoptosis 14: 536-548, 2009.

7. Kaufman RJ: Stress signaling from the lumen of the endoplasmic reticulum: coordination of gene transcriptional and translational controls. Genes Dev 13: 1211-1233, 1999.
8. Ron D: Translational control in the endoplasmic reticulum stress response. J Clin Invest 110: 1383-1388, 2002.

9. Ferri KF and Kroemer G: Organelle-specific initiation of cell death pathways. Nat Cell Biol 3: E255-E263, 2001.

10. Kaufman RJ: Orchestrating the unfolded protein response in health and disease. J Clin Invest 110: 1389-1398, 2002.

11. Xu C, Bailly-Maitre B and Reed JC: Endoplasmic reticulum stress: cell life and death decisions. J Clin Invest 115: 2656-2664, 2005.

12. Bernales S, Papa FR and Walter P: Intracellular signaling by the unfolded protein response. Annu Rev Cell Dev Biol 22: 487-508, 2006.

13. Wang XZ, Lawson B, Brewer JW, et al: Signals from the stressed endoplasmic reticulum induce C/EBP-homologous protein (CHOP/GADD153). Mol Cell Biol 16: 4273-4280, 1996.

14. Hetz C, Russelakis-Carneiro M, Maundrell K, Castilla J and Soto C: Caspase-12 and endoplasmic reticulum stress mediate neurotoxicity of pathological prion protein. EMBO J 22: 5435-5445, 2003.

15. Hotamisligil GS: Role of endoplasmic reticulum stress and c-Jun $\mathrm{NH} 2$-terminal kinase pathways in inflammation and origin of obesity and diabetes. Diabetes 54 (Suppl 2): S73-S78, 2005.

16. Okada K, Minamino T, Tsukamoto Y, et al: Prolonged endoplasmic reticulum stress in hypertrophic and failing heart after aortic constriction: possible contribution of endoplasmic reticulum stress to cardiac myocyte apoptosis. Circulation 110: 705-712, 2004

17. Thuerauf DJ, Marcinko M, Gude N, Rubio M, Sussman MA and Glembotski CC: Activation of the unfolded protein response in infarcted mouse heart and hypoxic cultured cardiac myocytes. Circ Res 99: 275-282, 2006.

18. Szegezdi E, Duffy A, O'Mahoney ME, et al: ER stress contributes to ischemia-induced cardiomyocyte apoptosis. Biochem Biophys Res Commun 349: 1406-1411, 2006.

19. White FC, Carroll SM, Magnet A and Bloor CM: Coronary collateral development in swine after coronary artery occlusion. Circ Res 71: 1490-1500, 1992.

20. Glembotski CC: Endoplasmic reticulum stress in the heart. Circ Res 101: 975-984, 2007.

21. Toth A, Nickson P, Mandl A, Bannister ML, Toth K and Erhardt P: Endoplasmic reticulum stress as a novel therapeutic target in heart diseases. Cardiovasc Hematol Disord Drug Targets 7: 205-218, 2007

22. Kitakaze M and Tsukamoto O: What is the role of ER stress in the heart? Introduction and series overview. Circ Res 107: 15-18, 2010.

23. Minamino T and Kitakaze M: ER stress in cardiovascular disease. J Mol Cell Cardiol 48: 1105-1110, 2010.

24. Herrmann JL: Do ameroid constrictors reliably occlude porcine coronary arteries? J Surg Res 161: 36-37, 2010.

25. Schroder M and Kaufman RJ: The mammalian unfolded protein response. Annu Rev Biochem 74: 739-789, 2005.

26. Wang XZ, Harding HP, Zhang Y, Jolicoeur EM, Kuroda M and Ron D: Cloning of mammalian Irel reveals diversity in the ER stress responses. EMBO J 17: 5708-5717, 1998.

27. Yoshida $\mathrm{H}$, Okada T, Haze $\mathrm{K}$, et al: ATF6 activated by proteolysis binds in the presence of NF-Y (CBF) directly to the cis-acting element responsible for the mammalian unfolded protein response. Mol Cell Biol 20: 6755-6767, 2000.

28. Zinszner H, Kuroda M, Wang X, et al: CHOP is implicated in programmed cell death in response to impaired function of the endoplasmic reticulum. Genes Dev 12: 982-995, 1998.

29. Oyadomari S, Koizumi A, Takeda K, et al: Targeted disruption of the Chop gene delays endoplasmic reticulum stress-mediated diabetes. J Clin Invest 109: 525-532, 2002.

30. Nakagawa $\mathrm{T}$, Zhu $\mathrm{H}$, Morishima $\mathrm{N}$, et al: Caspase-12 mediates endoplasmic-reticulum-specific apoptosis and cytotoxicity by amyloid-beta. Nature 403: 98-103, 2000.

31. Rao RV, Castro-Obregon S, Frankowski H, et al: Coupling endoplasmic reticulum stress to the cell death program. An Apaf-1-independent intrinsic pathway. J Biol Chem 277: 21836-21842, 2002.

32. Urano F, Wang X, Bertolotti A, et al: Coupling of stress in the ER to activation of JNK protein kinases by transmembrane protein kinase IRE1. Science 287: 664-666, 2000.

33. Davis RJ: Signal transduction by the JNK group of MAP kinases. Cell 103: 239-252, 2000. 